\title{
El prestigio frente a la identidad: las actitudes lingüísticas de los monterianos hacia el español hablado en Montería*
}

\author{
AURA SALAZAR CARO \\ aura01081@hotmail.com
}

Forma de citar este artículo: Salazar Caro, A. (2014). El prestigio frente a la identidad: las actitudes linguísticas de los monterianos hacia el español hablado en Montería. Cuadernos de Lingüística Hispánica, 25, 39-55. Tunja: Uptc.

* Artículo de investigación. Surge en el marco de una investigación sobre actitudes lingüísticas que vengo desarrollando desde el año 2011. Con este proyecto he participado en congresos nacionales (Montería, Tunja y Bogotá). Actualmente estoy dándole continuidad desde la Maestría en Investigación Social Interdisciplinaria (MISI) de la Universidad Distrital Francisco José de Caldas. El artículo deriva de una ponencia que presenté en octubre de 2013 en el Congreso Colombiano de Jóvenes Linguísticas, organizado por la Universidad Nacional de Colombia y el Instituto Caro y Cuervo.

** Licenciada en Lengua Castellana de la Universidad de Córdoba (Colombia). Estudiante de la Maestría en Investigación Social Interdisciplinaria (MISI) de la Universidad Distrital Francisco José de Caldas (Bogotá- Colombia). Investigadora social con énfasis en sociolingüística. 


\title{
Resumen
}

En este artículo se analizan aspectos como el grado de identidad y de lealtad de los monterianos para con su variedad, el dilema que surge entre los hablantes cuando se enfrentan a lo que creen que es lingüísticamente correcto y lo que sienten como culturalmente propio, y la actitud adoptada ante comunidades linguísticas que gozan de mayor prestigio. El estudio se centra en el componente emotivo de la actitud, por ser este el que incluye la valoración y evaluación de las variantes dialectales de la comunidad de habla. El análisis de los datos se realizó con base en las variables pre-estratificatorias sexogénero, nivel de instrucción y edad, propuestas por PRESEEA (Proyecto para el Estudio Sociolingüístico del Español de España y América). Los aspectos señalados al principio se abordan, inicialmente, en términos generales y luego se analizan a partir de las variables que resultaron más influyentes.

Palabras clave: actitudes lingüísticas, español hablado en Montería, identidad y prestigio.

\section{Prestige versus identity: the linguistic attitudes of people from Montería towards Spanish spoken in Montería.}

\begin{abstract}
This article analyzes aspects such as the degree of identity and loyalty of people from Montería towards their language variety, the dilemma arousing among speakers when dealing with what is believed to be linguistically correct and what is felt as culturally owned, and their attitude towards more prestigious linguistic communities. The study focuses on the emotional component of attitude, as it includes the assessment and evaluation of dialectal variations in the speech community. The data analysis was carried out according to the pre-stratified variables of sex-gender, level of education, and age proposed by PRESEEA (Proyect for the Sociolinguistic Study of Spanish in Spain and America). Initially, the aforementioned aspects are broadly discussed; then analyzed from those variables which turned out to be the most influential.
\end{abstract}

Key words: linguistic attitudes, Spanish spoken in Montería, identity and prestige. 


\section{Le prestige face à l'identité: les attitudes linguistiques des monterianos envers l'espagnol parlé à Montería.}

\section{Résumé}

Dans cet article on analyse des aspects tels que le degré d'identité et de loyauté des monterianos à l'égard de leur variété; le dilemme qui surgit entre les parlants quand ils confrontent ce qu'ils considèrent linguistiquement correcte et ce qu'ils sentent comme culturellement à eux, et l'attitude adoptée face aux communautés linguistiques qui ont un prestige majeur. L'étude se centre sur le composant émotif de l'attitude, car celui-ci inclue la mise en valeur et l'évaluation des variantes dialectales de la communauté de parole. L'analyse des données a été faite en ayant comme base les variables pré-stratifiées - sexe, genre, niveau de formation et âge - proposées par PRESEEA (Projet pour l'Étude Sociolinguistique de l'Espagnol de l'Espagne et des Amériques). Les aspects signalés au début, sont abordés initialement, en termes généraux et après ils ont analysés à partir des variables qui sont devenues plus influentes.

Mots clés: attitudes linguistiques, espagnol parlé à Monteria, identité et prestige.

\section{0 prestígio frente à identidade: as atitudes linguísticas dos monterianos a respeito do espanhol falado em Monteria (um estado da Colômbia).}

\section{Resumo}

Neste artigo se analisam aspectos como o grau de identidade e de lealdade dos monterianos para com sua variedade, o dilema que surge entre os falantes quando se enfrentam ao que creem que é linguisticamente correto e o que sentem como culturalmente próprio, e a atitude adotada perante comunidades linguísticas que gozam de maior prestígio. 0 estudo se centra no componente emotivo da atitude, por ser este o que inclui a valorização e a avaliação das variantes dialectais da comunidade de fala. A análise dos dados se realizou com base nas variáveis pre-estratificatórias sexo-gênero, nível de instrução e idade, propostas por PRESEEA (Projeto para o Estudo Sociolinguístico do Espanhol da Espanha e da América). Os aspectos assinalados ao princípio se abordam, inicialmente, em termos gerais e após, se analisam a partir das variáveis que resultaram mais influentes.

Palavras chave: atitudes linguísticas, espanhol falado em Monteria, identidade e prestígio. 


\section{Introducción}

Las nociones de prestigio e identidad resultan determinantes en cualquier estudio de actitudes lingüísticas, debido a que las consideraciones acerca de una lengua se relacionan con la posición de los grupos sociales que hacen uso de ella (Castillo, 2006, p. 296). Desde el punto de vista social, se reconoce el valor del prestigio que adquiere una variedad frente a otra, ya que las diferencias dialectales generan ciertas actitudes sociales que hacen que unas lenguas sean valoradas de forma positiva y otras de forma negativa. A continuación, se analizan algunos de los conflictos que existen entre los monterianos cuando se enfrenta la noción que tienen acerca de las hablas más prestigiosas, con el valor simbólico que le asignan a su variedad propia como elemento identitario.

Este artículo está centrado en analizar aspectos como el grado de identidad y de lealtad de los monterianos para con su variedad, el dilema que surge entre los hablantes cuando se enfrenta lo que consideran que es lingüísticamente correcto y lo que sienten como culturalmente propio, y la actitud adoptada frente a comunidades linguísticas que gozan de mayor estatus. Se analiza cómo es posible que aún cuando los monterianos consideran que su habla carece de prestigio lingǘstico y resulta inadecuada en campos como la educación y la política pública, en el componente emotivo prefieren reconocer el valor identitario de su variedad propia.

La tradición psicológica ha adoptado un modelo tríptico en el que las actitudes obedecen a una categoría cognitiva, a una categoría afectiva y a una categoría conativaconductual ${ }^{11}$. Estas tres vías explican, según Morales (1996), la complejidad interna de la actitud y el hecho de que muchos autores hablen de los tres componentes de la actitud. Este autor plantea que mientras en las respuestas cognitivas se tiene en cuenta la evaluación positiva o negativa de un objeto, producida a través de pensamientos e ideas,

1 El término conativo se asocia en psicología con lo conductual en la medida en que se refiere a todo aquello que es expresado en la conducta. Se refiere a las tendencias e inclinaciones del sujeto, por tanto abarca lo que las personas hacen, lo que dicen y lo que parece que harán. 
denominadas creencias, y en las afectivas se consideran los sentimientos, estados de ánimo y las emociones asociados con el objeto de la actitud, en las respuestas conativas o conductuales se tienen en cuenta tanto las conductas o actuaciones como las intenciones 0 conductas expresadas por el sujeto frente al objeto actitudinal.

El análisis se centra en el componente emotivo de la actitud, por ser este el que incluye la valoración y la evaluación de las variantes dialectales de la comunidad de habla; las emociones que inspira el objeto en la persona, sean éstas positivas o negativas; y los juicios de valor (estima-odio) acerca de las características del habla (variedad dialectal, acento), de la asociación de la identidad (etnicidad, lealtad, orgullo, valor simbólico) y del sentimiento de solidaridad con el grupo de pertenencia (Makuc, 2011; López Morales, 2004 y Blas Arroyo, 2002).

Considerando que la investigación de la que hace parte esta propuesta acoge la metodología del proyecto PRESEEA ${ }^{2}$, el análisis de los datos se hace con base en las variables pre-estratificatorias sexo- género, nivel de instrucción y edad ${ }^{3}$. Los aspectos señalados al principio se abordan inicialmente en términos generales y luego se analizan con base en las variables extralingüísticas que resultaron más influyentes dentro de cada uno de los tópicos estudiados. El estudio de estas variables se justifica precisamente porque las actitudes varían dependiendo de factores como la edad, el género, el estatus social y el nivel de formación académica (Hernández- Campoy [s.f]).

\section{Actitud, prestigio e identidad}

Álvarez y Medina (2001) señalan que el problema de las actitudes lingüísticas hacia ciertas variedades se relaciona con el prestigio que estas tienen ante sus hablantes. Por ello, es necesario aclarar, primero, la noción de prestigio lingüístico y, segundo, las relaciones que este tiene con el concepto de corrección. Con respecto a la primera cuestión, Hernández- Campoy [s.f]) señala que:

El prestigio en Sociolingüística es un comportamiento lingüístico motivado por las actitudes sociales adoptadas ante determinadas formas linguísticas que se refieren a la estima que adquieren algunas variedades dialectales, acentos o incluso rasgos

2 PRESEEA es un proyecto de la Comisión de Sociolingüística de ALFAL (Asociación de Lingüística y Filología de la América Latina), que surge en 1993 con el objetivo de coordinar las investigaciones sociolinguísticas de Iberoamérica y de la Península Ibérica, para facilitar la comparabilidad de los estudios y el intercambio de información (PRESEEA,2003). La sede central de PRESEEA se encuentra en la Universidad de Alcalá de Henares (España). El proyecto ofrece una metodología que resulta útil para estudios sociolinguísticos, en la medida en que ofrece pautas específicas en cuanto a la selección de la muestra y la recogida de los materiales.

3 Tomando dos generaciones: I (de 20- 35 años) y 2 (de 55 años en adelante). 
lingüísticos determinados, como consecuencia de una reputación adquirida 0 atribuida, que es totalmente subjetiva (p. 41).

Cuando el autor define el prestigio como la estima que adquieren algunas variedades, deja claro que se trata de un factor adquirido, no intrínseco. Por ello es que resulta difícil determinar si la actitud manifestada es hacia fenómenos lingüísticos específicos o si se trata de una valoración hacia los usuarios de un determinado grupo social (López Morales, 2004). El prestigio obedece a una naturaleza eminentemente social debido a que son los sujetos quienes se lo atribuyen a las variedades o usos lingüísticos empleados por grupos prestigiosos (Hernández- Campoy, [s.f]).

El carácter social del prestigio permite que sean los hablantes quienes establezcan los parámetros para posicionar una variedad, considerada por ellos "correcta", por encima de otra, considerada "incorrecta" (Hernández- Campoy, [s.f]). Al respecto, Trudgill (1983) señala que el estudio científico de la lengua ha demostrado que todos son igualmente 'buenos' como sistemas lingüísticos. Según él, todas las variedades de una lengua son complejos sistemas estructurados, gobernados por reglas y completamente adecuados a las necesidades de sus hablantes, por lo que señala:

Los juicios de valor referidos a la corrección y pureza de las variedades lingüísticas son sociales más que lingüísticos. No hay nada en absoluto que sea inherente a las variedades no estándares y que las haga inferiores. Cualquier inferioridad aparente sólo se debe a su relación con hablantes pertenecientes a grupos no privilegiados y de bajo estatus (p. 20).

De acuerdo con esto, un dialecto puede ser considerado "más correcto" que otro sencillamente porque es hablado por un grupo socialmente prestigioso y esa valoración la hacen los mismos hablantes, debido a que como señala Hernández- Campoy [s.f]), lingüísticamente hablando, ninguna variedad podría estar por encima de otra. De hecho, las variedades no estándares son consideradas inferiores a las estándares precisamente porque, al ser usadas por grupos no prestigiosos, cuentan con un menor estatus social.

Álvarez y Medina (2001) señalan que la imagen que los hablantes tienen de sí mismos se acerca muchas veces más a una norma de corrección que a la realidad. Según estas autoras, en lo que concierne a la variedad que las personas hablan, algunos estudios (cf.Giles, 1979) muestran que, para asegurar su aceptación por el grupo, la gente manifiesta su preferencia por las variedades de prestigio, es decir, por aquellas variedades lingüísticas más cercanas a la norma.

Las autoras plantean que los hablantes confiesan su preferencia por los dialectos de mayor estatus, aún cuando ellos mismos no los hablen. Sin embargo, hay que señalar 
que hay un punto en el que el prestigio se ve enfrentado con la identidad, precisamente porque las variedades propias pueden ser vistas de forma favorable. Ellas indican esto:

La separación entre el poder y el prestigio se profundiza cuando se refiere a los temas relacionados con el dialecto y la identidad, debido al hecho de que la gente puede reconocer la variedad de prestigio y, sin embargo, defender su identidad regional. De esto se deriva que, en cualquier país, la capital es el domicilio de los poderes públicos y probablemente también la región donde se habla la variedad lingüística de prestigio (p. 3).

Es evidente que la variedad propia puede ser evaluada de forma positiva, aunque no posea ni prestigio ni un alto nivel de estandarización, si los hablantes la reconocen como un elemento que define su pertenencia a determinada región. En este sentido, podría definirse la identidad cultural como un factor determinante en la construcción de la actitud de los hablantes hacia la variedad propia. Asencio (2009) concuerda con Saville-Troike (2001) cuando afirma que las actitudes positivas acerca de la lengua propia son normalmente generadas por el rol que juega como símbolo de la identidad del grupo y crea sentimientos negativos cuando tal identidad es rechazada.

Como se observa, el grado de identidad de los hablantes puede generar actitudes positivas o negativas hacia la variedad lingüística propia. Si los hablantes se sienten identificados con su dialecto, es probable que le tengan un gran aprecio; pero si no lo conciben como un elemento identitario, es menos probable que lo vean de forma favorable.

\section{Las actitudes lingüísticas de los monterianos: cuestiones metodológicas}

Gómez (2001, p. 25) considera que "una de las premisas fundamentales en sociolingüística es aplicar la metodología más idónea a los fines de la investigación". Por ello, una de las principales preocupaciones del sociolingüista es aplicar los métodos y las técnicas que sean coherentes con los objetivos propuestos. El tipo de métodos que utilicen los estudios elaborados con base en cierta perspectiva teórica, dependerá tanto de la visión que se tenga acerca del fenómeno, como de los elementos que el investigador considere que presentan relevancia en el proceso (Sánchez, 2005).

\subsection{Tipo de estudio}

Hernández Campoy (s.f.) señala que el estudio de las actitudes, como cualquier estudio empírico experimental, sigue un proceso de formulación de hipótesis mediante el procedimiento hipotético-deductivo y contrastación mediante la observación y la experimentación. Según el autor, aunque este suele ser más el protocolo de la metodología cuantitativa, la metodología cualitativa también resulta muy útil. 
De acuerdo con lo anterior, y en consonancia con autores como Flick (1995) y Ortí (1995), referenciados por Almeida y Hernández (2005), que defienden la complementariedad de la metodología cualitativa y cuantitativa; en este estudio se ofrece de forma conjunta un análisis cuantitativo y cualitativo de los datos obtenidos. Silva-Corvalán (2001) aclara que la sociolingüística no es una ciencia exclusivamente positivista.

Como bien señala Hernández Campoy (s. f), el método cuantitativo permite expresar de modo rigurosamente preciso y claro los resultados de la investigación. Por ello, a fin de dar cuenta de un fenómeno tan complejo como el de las actitudes lingüísticas, fue necesario complementar el lenguaje matemático y lógico propio de la metodología cuantitativa con las opiniones explícitas de los mismos informantes.

\subsection{El estudio de las actitudes: el método directo}

Izquierdo (2003: 20) señala que "el estudio de las actitudes lingüísticas se ha planteado desde dos perspectivas distintas, si bien con resultados que pueden ser complementarios: la mentalista y la conductista". El presente estudio se inscribe en el enfoque mentalista, considerando que, de acuerdo con Blas Arroyo (1999), "la mayor parte de los investigadores en el terreno de las actitudes lingüísticas se han adherido a este punto de vista”. Lo que interesa averiguar es, como bien lo señala Moreno (1998), la disposición mental de los hablantes.

Con respecto a la metodología que debe emplearse en este modelo, Erdösová (2011: 5) señala que desde el punto de vista mentalista "se plantea el objetivo de capturar un modo de pensar, no necesariamente de actuar". En este sentido, el método y las técnicas empleadas deben ser coherentes con este propósito.

Con respecto al método, hay que señalar que se distinguen dos tipos fundamentales de medición de las actitudes: la directa, que se lleva a cabo mediante entrevistas 0 cuestionarios sobre aspectos lingüísticos específicos; y la indirecta, que suele realizarse mediante la llamada técnica mached-guise (Almeida y Hernández-Campoy, 2005 citando a Ryan, Giles y Hewstone, 1988).

En este proyecto, la medición de las actitudes se hizo a través del método directo. Castillo (2006) señala que en la medición directa se pide a los hablantes que den su opinión sobre la lengua y en la indirecta se evita que el hablante se entere de que se trata de estudiar sus actitudes ante la lengua. Dentro de las técnicas de las mediciones directas, el autor resalta los cuestionarios con preguntas abiertas o cerradas, las entrevistas y la observación. Drevdal (2009: 33) señala que en el método directo, la obtención de datos se basa en las respuestas de un grupo de informantes a unas preguntas directas. 
Erdösová (2011) afirma que a los métodos directos pertenecen los cuestionarios de preguntas tanto abiertas como cerradas, los que siguen siendo el método más empleado en la investigación actitudinal; y las entrevistas. De acuerdo con González (2010), los cuestionarios se emplean en el método directo porque permiten una aproximación a opiniones y creencias muy reveladoras acerca del contexto sociolingüístico en el que se desenvuelve cada comunidad.

De las técnicas señaladas por los autores, en este estudio se empleó una entrevista semiestructurada en la que se incluyó un cuestionario con preguntas cerradas. Como bien señalan Álvarez, Martínez y Urdaneta (2001), un método directo pediría a los informantes que respondieran a un cuestionario escrito o a las preguntas de una entrevista, donde se les pide directamente dar su opinión sobre una u otra lengua.

\subsection{Selección de los hablantes}

Para la selección de los hablantes y la recogida de la muestra se acogió la metodología propuesta por PRESEEA. La muestra total fue de 24 hablantes nativos, considerando que en los núcleos con un número de habitantes inferior a 500.000 habitantes es posible reducir el número de informantes por cuota, lo que daría una muestra de 54 hablantes. Esta muestra resulta representativa si se considera: primero, que según datos del DANE para 2012, Montería tiene una población total de 422.175 habitantes, de los cuales 324.711 viven en el casco urbano y 97.464 en el área rural; y segundo, que en esta investigación se trabaja con dos generaciones.

Sinave (2009) considera que al existir diferencias marcadas en las hablas de las diferentes generaciones, se puede suponer que lo mismo ocurre en cuanto a las actitudes de estas. El estudio de las generaciones 1 y 3 permite confrontar las actitudes de uno y otro grupo y establecer posibles diferencias respecto a los usos de determinados rasgos lingüísticos según cada generación.

\section{Análisis de datos}

\subsection{El español de Montería como elemento identitario}

El grado de identidad de los monterianos para con su variedad fue una de las cuestiones fundamentales que se incluyó dentro del componente emotivo. La primera pregunta ¿Usted se siente identificado con la forma de hablar de los monterianos? se orienta a saber si los hablantes se sienten o no identificados con el español que hablan. 
Gráfico 1: ¿Usted se siente identificado con la forma de hablar de los monterianos?

因 血No 目un poquito/ en algún sentido $\otimes N S / N R$

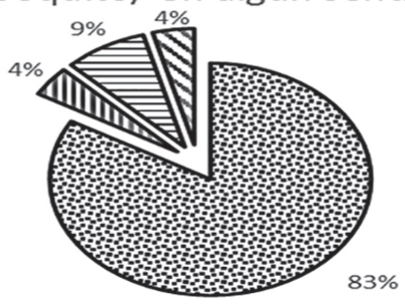

En el componente cognitivo, la concepción más predominante entre los monterianos acerca de su habla es aquella que la define como señal de identidad de un pueblo. Aunque en el componente cognitivo, el 63\% definió el español hablado en Montería como un elemento que identifica a un pueblo, cuando se apeló al grado de identificación personal del hablante con su variedad, resultó que el 83\% afirmó sentirse identificado con su habla propia. Queda claro, entonces, que entre los monterianos el habla propia constituye un rasgo fundamental de su configuración identitaria.

Álvarez, Martínez y Urdaneta (2001) definen la identidad como una imagen de sí mismo que es corroborada o no por los demás y que suscita una respuesta emocional, que puede ser positiva o negativa. En este sentido, el grado de identidad de los hablantes con su variedad depende de las emociones que esta les inspira. Puede ser que entre los monterianos, al haber un alto grado de identificación, predominan los sentimientos de lealtad para con la variedad propia o que exista un arraigo cultural que los lleva a considerar que la variedad que hablan los diferencia de otros pueblos y los posiciona en cierto espacio cultural que sienten como autóctono.

\subsubsection{Identidad y lealtad hacia el español hablado en Montería}

En la segunda pregunta Si pudiera cambiar su manera de bablar, ¿qué acento le gustaría tener?, se intentó averiguar si el grado de identidad de los hablantes para con su variedad influye en el grado de lealtad de estos frente a su propia habla. Por ello, se incluyeron aquí aspectos relacionados con la posibilidad de cambiar de variedad y el comportamiento lingüístico que reflejarían los hablantes al interactuar con variedades lingüísticas ajenas. 
Gráfico 2: Si pudiera cambiar su manera de hablar, ¿qué acento le gustaría tener?

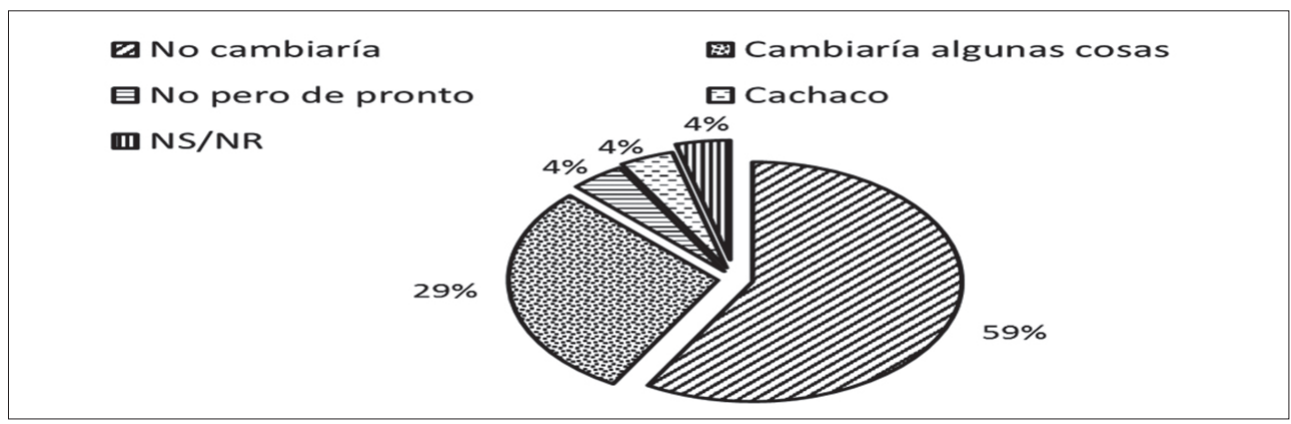

Drevdal (2009, p. 15) afirma que "una actitud positiva y una identificación fuerte con la propia variedad linguística hace que la persona sienta un alto nivel de lealtad hacia esa variedad". Es interesante resaltar aquí que el 59\% de los hablantes no considera siquiera la posibilidad de cambiar su variedad por otra. Tanto en el componente cognitivo como en el tópico anterior, quedó claro que los monterianos tienen un alto grado de identificación con la variedad lingüística propia. Como señala el autor, el grado de identidad hace mucho menos probable que el hablante cambie drásticamente su forma de hablar.

\section{Variable edad}

En el gráfico 3 queda claro que existen diferencias entre el grado de lealtad de la generación 1 frente a la 3. Mientras en la generación 3 un 83\% de los sujetos reconoce que no cambiaría su variedad, en la generación 1 sólo un 33\% manifiesta lo mismo. Es evidente además que quienes se enfrentan al dilema entre lo correcto o prestigioso y lo identitario son los hablantes de la generación 1. El 50\% de los hablantes de esa generación consideran que aunque sería desleal cambiar su variedad, sí es posible cambiarle algunas cosas.

Drevdal (2009) señala que si la actitud de los hablantes es positiva y hay un alto grado de identidad, habrá una mayor lealtad con el grupo; por ello, el hecho de que entre las generaciones mayores haya un grado de identificación más fuerte para con el español hablado en Montería, hace que sea menos probable que los hablantes de esta generación cambien su variedad o que terminen adoptando formas lingǘsticas ajenas a la suya. 
Gráfico 3: Si pudiera cambiar su manera de hablar, ¿qué acento le gustaría tener?/ edad

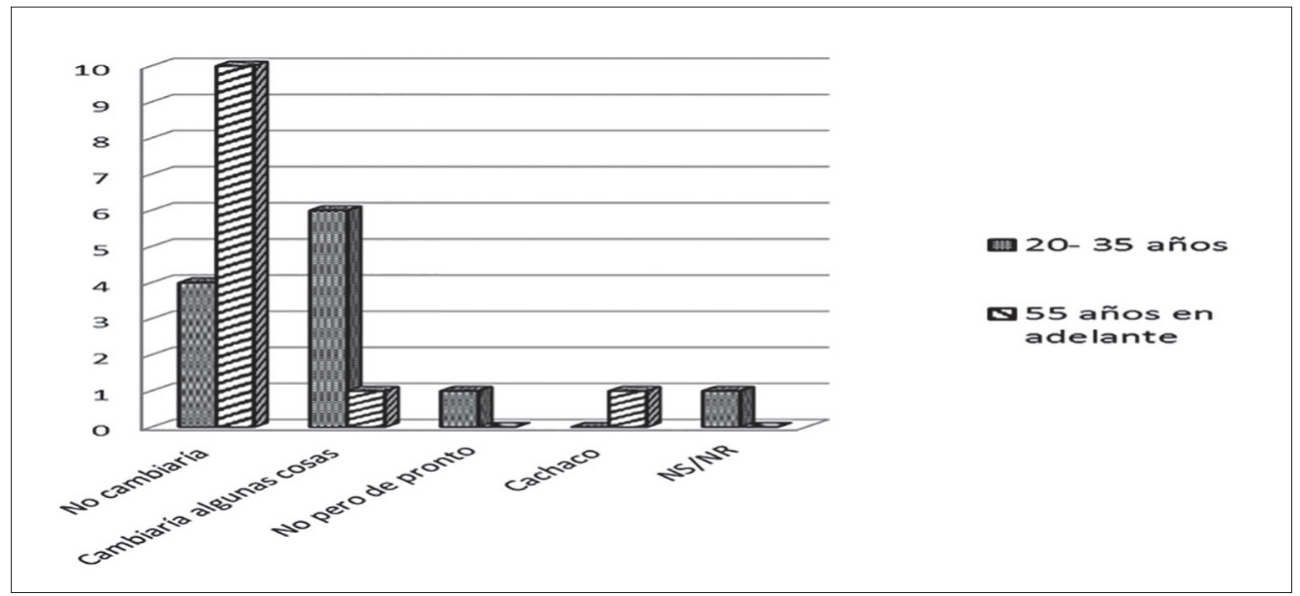

\subsubsection{Identidad versus prestigio}

En el componente cognitivo los monterianos reconocieron que Bogotá y Medellín son las regiones en las que "se habla mejor". En el componente emotivo se les preguntó Si usted viajara boy a Bogotá para estar allá unos años, ¿cómo hablaría: como hablan aquí o como bablan allá?, con la intención de enfrentar sus nociones de identidad y prestigio. Se referencia a Bogotá porque esta es la ciudad capital de la República de Colombia, y el epicentro de las ramas del poder público. Álvarez, Martínez y Urdaneta (2001) consideran que en la mayoría de los estudios sobre actitudes se desprende que el dialecto de la región que ostenta el poder político es también el dialecto de mayor estatus social. Por tanto, al ser Bogotá el centro del poder político es también una de las regiones colombianas con mayor prestigio lingüístico.

Gráfico 4: Si usted viajara hoy a Bogotá para estar allá unos años, ¿cómo hablaría: como hablan aquí o como hablan allá?

\section{Igual que aquí}

Igual que ellos

푸 Trataría de hablar moderadamente

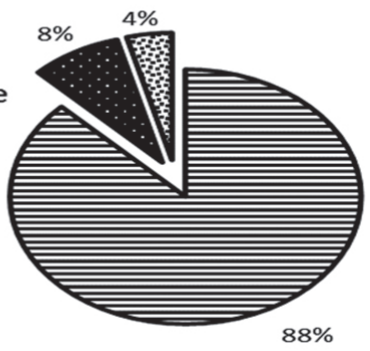


Como puede verse, el 88\% de los informantes afirma que si estuviese en Bogotá hablaría como habla en Montería. Este porcentaje revela que aunque los hablantes reconocen el habla bogotana como una variedad más prestigiosa que la suya, manifiestan que muy por encima del prestigio, está la identidad. Hay que recordar que, de acuerdo con Medina (2001), la gente puede defender su identidad regional por encima de cualquier otra cosa y esto hace que, a la hora de decidir cuál sería la actitud adoptada al internarse en una comunidad lingüística de mayor prestigio, los monterianos manifiesten abiertamente que continuarían hablando como hablan en su ciudad de origen.

A la pregunta ¿Qué opina de las personas que viajan a Bogotá o a Medellín y se les pega la manera de hablar de allá?, el 33\% de los hablantes manifiesta que los monterianos que viajan a Bogotá y se les pega la manera de hablar de los bogotanos es porque no tienen identidad cultural, y otro $33 \%$ considera que eso es corroncheh, pendejá o maricát . Estos porcentajes, que además son los mayores, demuestra la tendencia de los monterianos a relacionar la variedad propia con la identidad cultural. Los dos últimos vocablos calificativos, además, demuestran el desacuerdo de los monterianos con los hablantes que adoptan las formas de hablar del interior del país.

\section{Gráfico 5: ¿Qué opina de las personas que viajan a Bogotá o a Medellín} y se les pega la manera de hablar de allá?

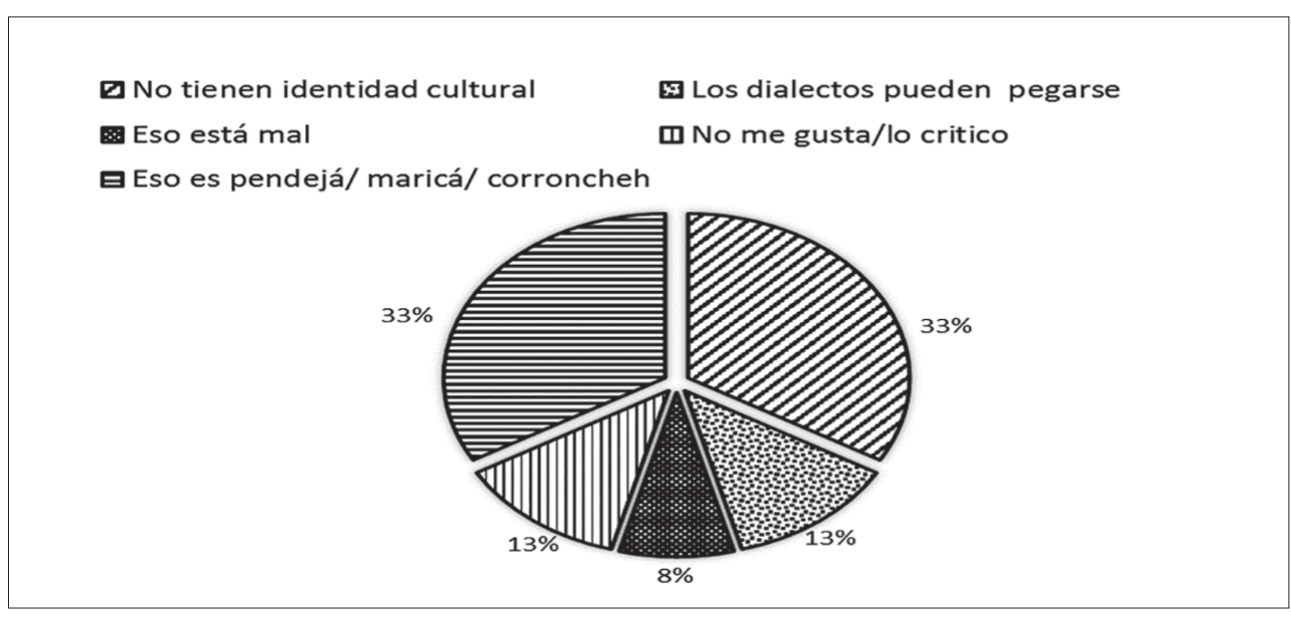

4 Corroncheh es un término que emplean en Montería y en general en la Costa Caribe para referirse a una acción o evento que demuestra falta de identidad, de formación o de cultura. Los términos pendejá o maricá se usan para calificar acciones injustificadas que son consideradas como bobadas o pretensiones. En este estudio se hizo una transcripción fonética de las entrevistas, por ello trato de ser fiel a las elisiones y a las aspiraciones hechas por los informantes. 
En comentarios como digo que debemoh mejorar / de pronto ciertab condiciones del hablado/pero no cambiar (CAST0133M) ${ }^{5}$, poqque /si me entiendeh/ uno cuida lab raiceb (CENT01321H), a mi me da eb como rabial o pena con esa persona poqque cuando vienen acá eb pa' que lob demáb se bullen de ellob (GRAN02023M), los hablantes no sólo reconocen que conservar la forma de hablar propia es una forma de cuidar las raíces, los orígenes; sino que reconocen que emplear variedades del interior del país es exponerse a la burla y a la vergüenza pública.

\section{Variable nivel de instrucción}

González (2003) considera que uno de los factores que más influye en el comportamiento lingüístico de los hablantes es el grado de instrucción que poseen. Los datos aquí reseñados demuestran que el nivel de instrucción no solo influye en la actuación lingüística, sino también en la actitud lingüística de los monterianos. Como puede verse en el gráfico, entre los profesionales se observa una clara tendencia a relacionar la adopción de una variedad ajena con la falta de identidad. El 75\% de los monterianos bachilleres considera que el monteriano que viaja a Bogotá o a Medellín y adopta las formas lingüísticas de esas ciudades carece de identidad.

Gráfico 6: ¿Qué opina de las personas que viajan a Bogotá o a Medellín y se les pega la manera de hablar de allá?/ nivel de instrucción

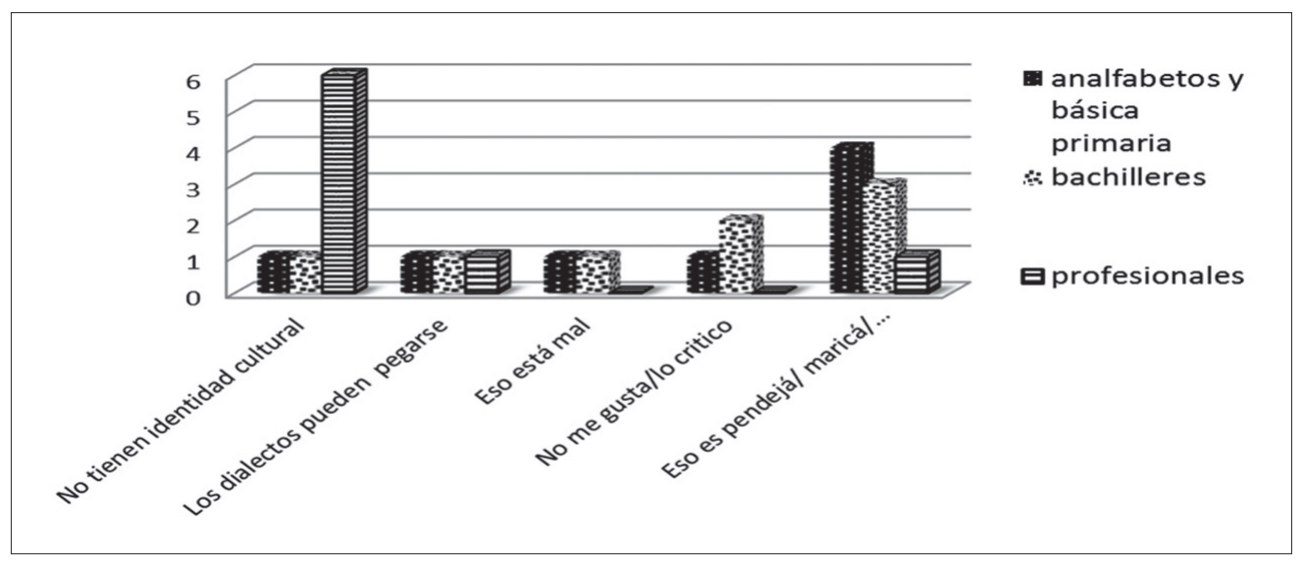

5 A cada uno de los informantes se les asignó un código de acuerdo con su caracterización social tal como se indica a continuación: identificación del código: CAST133M//CAST: Barrio, La Castellana //01: número del orden del informante//3: grado de instrucción: estudios superiores//3: edad/ 55 en adelante//M: sexo, mujer. Identificación del código CENT01321H// CENT: Barrio, El Centro//013: número del orden del informante//2: grado de instrucción /: bachiller//1: edad/ 20- 35 años//H: sexo, hombre. Identificación del código GRAN02023M// GRAN: Barrio, La Granja//020: número del orden del informante//2: grado de instrucción /: bachiller//3: edad/ 55 años en adelante//M: sexo, mujer. 
También hay que resaltar que entre los analfabetas, la tendencia es a considerar que se trata de una pendejá, una maricá, propia de un corrocho. Los términos empleados, en sí mismos, evidencian hasta qué grado pueden comprometerse las emociones de los hablantes a la hora de enfrentar la deslealtad al grupo por parte de uno de sus miembros que adopta una variedad lingüística ajena.

\section{Discusión}

Es evidente que a pesar de que en el componente cognitivo los hablantes definen su variedad como señal de identidad, aun cuando reconocen que carece de prestigio lingüístico y que resulta inadecuada en campos como la educación y la política pública, en el componente emotivo, llegado el punto en el que los sentimientos de identidad se enfrentan con el prestigio lingüístico, se nota una clara preferencia por el reconocimiento del valor identitario de la variedad propia. Hay que señalar que los casos en los que se pone a prueba la lealtad del hablante, como el de los informantes que señalan que no cambiarían su variedad, pero sí le cambiarían algunas cosas, constituyen claros ejemplos de cómo el componente cognitivo puede terminar enfrentándose con el componente emotivo, al poner a los hablantes a elegir entre lo que socialmente consideran prestigioso y lo que emocionalmente sienten como propio.

\section{Referencias bibliográficas}

Almeida, M., \& Hernández, J. (2005). Metodología de la investigación sociolingüística. Málaga: Comarca.

Álvarez, A., Martínez, H., \& Urdaneta, L. (2001). Actitudes lingüísticas en Mérida y Maracaibo: Otra cara de la identidad. Boletín Antropológico, 2(52), 145-166. Mérida: Universidad de Los Andes. Recuperado de http://webdel profesor.ula.ve/humanidades/hernan. martinez/publicaciones/Actitudes\%20ling\%FC\%EDsticas\%20en\%20habitantes\%20de\%20 M\%E9rida\%20y\%20Zulia.pdf

Álvarez, A., \& Medina, A. (2001). Actitudes lingüísticas en adolescentes andinos En: Domínguez, C., Pietrosemoli, L. y Álvarez, A (eds.) Homenaje a Paola Bentivoglio. Estudios lingüísticos. Universidad de Los Andes: Cuadernos de Lengua y Habla, pp. 29-50.

Asencio, M. (2009). La pérdida de una lengua: el caso del Náhuat. Teoría y praxis, (14). San Salvador: Universidad do Bosco. Recuperado de http://www.udb.edu.sv/editorial/pdf/ revistas/teoriaypraxis/n14/4.\%20La\%20perdida\%20de\%20una\%20lengua\%20El\%20 Caso\%20del\%20Nahuat.pdf 
Blas Arroyo, J. (1999). Las actitudes hacia la variación intradialectal en la sociolingüística hispánica. Estudios Filológicos, 34, 44-72. Recuperado de http://www.uv.es/socant2/salamlleng.pdf

Blas Arroyo, J. (2002). Lenguas en contacto y actitudes lingüísticas en la comunidad de Valencia. En: Blas Arroyo, J. (2002). Estudios sobre lengua y sociedad. Recuperado de http://books. google.com.co/ Recuperado de http://books.google.com.co/books?id=PzDimeUxlOwC\& $\mathrm{pg}=\mathrm{PA} 57 \& \mathrm{dq}=$ componentes $+\mathrm{de}+\mathrm{la}+\mathrm{actitud}+$ linguistica\&hl=es\&sa=X\&ei=4ubgT $57 \mathrm{CA} 4$ qu8AS245CHDQ\&ved=0CGYQ6AEwCQ\#v=onepage\&q=componentes\%20de\%201a $\% 20$ actitud\%20linguistica\&f=false

Castillo, M. (2006). El estudio de las actitudes lingüísticas en el contexto sociocultural: el caso del mexicano de Cuetzalan. Anales de antropología, 40, 1.

Drevdal, S. (2009). Actitudes lingüisticas de los inmigrantes nicaragüenses en Costa Rica bacia su propia habla. Tesis de Licenciatura, Universitetet i Bergen, Noruega. Recuperado de: https://bora.uib.no/bitstream/1956/3822/1/56492314.pdf.

Erdösová, Z. (2011). El español de México en los ojos de sus hablantes: un estudio desde la sociolingüística y la dialectología perceptiva. Lengua y voz, 1(1), 57-81. Recuperado: http://www.uaemex.mx/lenguayvoz/Revista/1/Articulos/El_esp_de_Mex_en_los_ojos_de_ sus_hablantes.pdf

Flick, U. (1998). An introduction to quality research. Londres: Sage.

Giles, H. (1979). Ethnicity markers in speech. En Scherer, Klaus y Howard Giles. Social markers in Speech. Cambridge: Cambridge University Press.

Gómez, J. (Coord.).(2001). El español hablado en Valencia. Materiales para su estudio (PRESEEA). Nivel sociocultural alto. Valencia: Universitat de Valencia.

González, J. (2010). Actitudes lingüísticas en una comunidad rural: Els Ports (Castellón). Datos de un cuestionario sociolingüístico. Cultura, Lenguaje y Representación, 8, 75- 95. Castellón: Universitat Jaimet I. Recuperado de http://www.e-revistes.uji.es/index.php/clr/ article/viewFile/64/62

Hernández- Campoy, M. (s.f). El fenómeno de las actitudes y su medición en sociolingüística. Universidad de Murcia. Recuperado de: http://www.um.es/tonosdigital/znum8/portada/ monotonos/04-JMCAMPOYa.pdf

Izquierdo, S. (2003). Actitudes ante el deterioro de la lengua. El español en Brasil. Madrid: Universidad de Alcalá. Recuperado de http://marcoele.com/descargas/13/izquierdodeterioro_de_la_lengua.pdf

López, H. (2004). Sociolingüística. Madrid: Gredos. 
Makuc, M. (2011). La actitud lingüística en la comunidad de habla de Magallanes: aproximación a sus componentes básicos. Magallanía, 39 (2), pp. 105-111.

Ortí, A. (1995). La confrontación de modelos y niveles epistemológicos en la génesis e historia de la investigación social. En: Delgado, J., \& Gutiérrez, J. (Comp.) (1995). Métodos y Técnicas Cualitativas de Investigación en Ciencias Sociales. Madrid: Síntesis. 85-95.

PRESEEA (Proyecto para el Estudio Sociolingüístico del Español de España y América), 2003. Metodología del "Proyecto para el Estudio Sociolingüístico del Español de España y de América. Recuperado de http://www.linguas.net/LinkClick. aspx?fileticket=\%2FthWeHX0AyY\%3D\&tabid=474\&mid $=928$

Sánchez, L. (2005). Los estudios sobre actitudes lingüísticas: panorama general, importancia, enfoques y propuesta de clasificación (tipología). Tesis de Licenciatura en Lingüística. Ciudad de México: Universidad Autónoma Metropolitana. Recuperado de http://tesiuami.izt. uam.mx/uam/aspuam/presentatesis.php?recno=12541\&docs=UAMI12541.pdf

Saville-Troike, M. (2003). The ethnography of communication: an introduction, tercera edición. Malden, MA: Blackwell Publishers Ltd.

Silva- Corvalán, C. (2001). Sociolingüística y pragmática del español. Washington: Georgetown University Press.

Sinave, N. (2009). Análisis de las actitudes lingüísticas hacia el slang mexicano: usos y valoración de la palabra gïey. Tesis de Maestría. Quebec: Universidad de Montréal. Recuperado de https://papyrus.bib.umontreal.ca/xmlui/bitstream/handle/1866/4296/ Sinave_Naila_2010_;jses sionid=A3ADCACEC24ACFE6F96791084D98E622? sequence $=4$

Trudgill, P. (1983) Sociolinguistics: An Introduction to Language and Society. London: Penguin (Revised Edition; 1974 First Edition). 